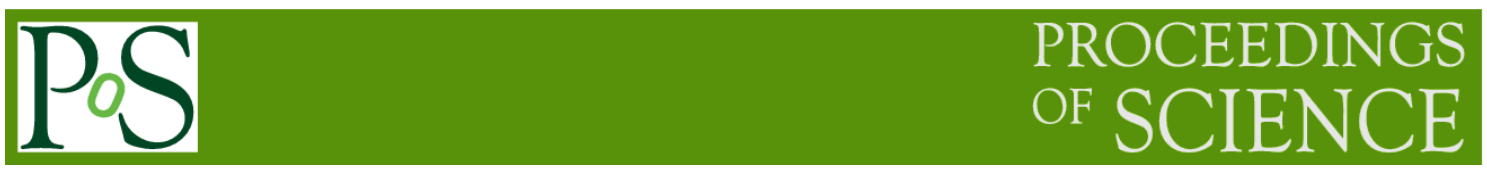

\title{
Study of Polystyrene Scintillators-WLS Fiber Elements and Scintillating Tile-WLS Prototypes for New CHOD Detector of CERN NA-62 Experiment
}

\author{
Vitaliy Semenov ${ }^{a}{ }^{1}$, Valery Brekhovskih ${ }^{a}$, Aleksandr Gorin ${ }^{a}$, Aleksey Khudyakov ${ }^{b}$, \\ Vladimir Rykalin ${ }^{a}$, Oleg Yushchenko ${ }^{a}$ \\ ${ }^{a}$ State Research Center of Russian Federation, Institute for High Energy Physics (IHEP), \\ Research Centre Kurchatov Institute \\ 1 Nauki sq, Protvino, Moscow region, 142281, Russia \\ ${ }^{b}$ Institute for Nuclear Research of the Russian Academy of Sciences (INR RAN) \\ 60-letiya Oktyabrya prospekt 7a, Moscow, 117312, Russia \\ E-mail: Vitaliy. Semenoveihep.ru
}

We measured the light output and the time resolution of few sets comprised of polystyrene scintillator and wavelength shifting (WLS) fibers as readout. The samples of different thickness $(7-30 \mathrm{~mm})$ have been made in the shape of bricks and plates with the areas of $25 \times 80,108 \times 134$ and $108 \times 268$ of $\mathrm{mm}^{2}$. In addition to samples of "ordinary" scintillator with additions of $2 \% \mathrm{p}$ Terphenyl $+0.05 \%$ POPOP, the rapid ultraviolet scintillator with single $2 \%$ additive PBD was tested. For the light collection WLS-fibers BCF92, Y11 and scintillation fiber SCSF-78M as reemitting were checked. The fibers were glued into the grooves on the front surface of scintillators. As the photo detectors silicon photomultipliers (SiPM) produced by CPTA (Russia) and SensL (Ireland) were used. It is shown that the dependence of light output on the thickness of scintillator is nonlinear and close to the square root function, which is also confirmed by the calculations carried out by Monte Carlo. The measured value of a light output makes it possible to obtain time resolution of about $0.5 \mathrm{~ns}$. The best result $(0.22 \mathrm{~ns})$ of the time resolution with the MIP detection is obtained for the pair of ultraviolet scintillator and SCSF-78M fiber.

International Conference on New Photo-detectors

PhotoDet 2015

6-9 July 2015

Moscow, Troitsk, Russia

\section{${ }^{1}$ Speaker}




\section{Introduction}

The main goal of NA62 experiment [1] is to measure precisely the branching ratio of the very rare decay $K^{+} \rightarrow \pi^{+} v \bar{v}$. NA62 plans to improve the current experimental precision exploiting a novel decay-in-flight technique based on calorimetry to veto extra particles, light mass trackers to reconstruct $K^{+}$and the $\pi^{+}$momenta and full particles ID capability. Expected intensity of hadron beam is $800 \mathrm{MHz}$ with $10 \mathrm{MHz}$ rate of decaying $K^{+}$. From the experimental point of view, the only one detected particle in the final state makes the identification of the rare decay extremely complicated. Therefore, for decay selection and background suppression a dedicated multi-level trigger system is developed.

The New Charged-particle Hodoscope (New CHOD) was designed as a pad structure detector allowing working in high intensity beam. This detector provides good time resolution at high counting rate [2]. The expected total rate of New $\mathrm{CHOD}$ was obtained from $\mathrm{MC}$ simulation and is expected to be of $35 \mathrm{MHz}$, with maximum rate per tile (around $500 \mathrm{Khz}$ ) in the region close to the beam pipe. It will be located at the end of the setup after the Ring Image Cherenkov (RICH) detector to trigger particles in the aperture in the Level-0 trigger and to suppress possible interactions in the RICH which will be used as a timing detector for single track events. New CHOD is covering the area of $2140 \mathrm{~mm}$ diameter with a hole of $280 \mathrm{~mm}$ diameter around beam pipe. The pad structure consists of 148 scintillating counters (tiles) with the size of $268 \times 108 \mathrm{~mm}^{2}$ (104 tiles) on periphery and $134 \times 108 \mathrm{~mm}^{2}$ (44 tiles) at the central region. Each tile has the dual SiPM readout. Thus, 296 electronic channels are required.

\section{The tile design.}

Each tile is made of polymerized scintillator produced at IHEP [3] with the thickness of $30 \mathrm{~mm}$ along the beam direction. The production of polystyrene scintillators was started at IHEP about 30 years ago. The large scintillator block thermal polymerization technology with following machining and polishing is used. The rectangular grooves cut by milling machine with the size of $1.1 \times 1.2 \mathrm{~mm}^{2}$ and $16.75 \mathrm{~mm}$ step are located on the top surface of the scintillator. Scintillation light is collected by $1 \mathrm{~mm}$ diameter WLS fibers glued into grooves. The WLS-fibers of different lengths from $1000 \mathrm{~mm}$ on the periphery to $2250 \mathrm{~mm}$ for the central part are grouped into two bundles (Fig. 1).

\section{MicroFC-30035-SMT-WP}

[4] silicone photomultipliers (SiPM) with the area of $3 \times 3$ $\mathrm{mm}^{2}$ (SensL, Ireland) are used.

The tiles were tested in a beam at CERN using 7 standard prototypes and 10 half-size prototypes. Encouraging results

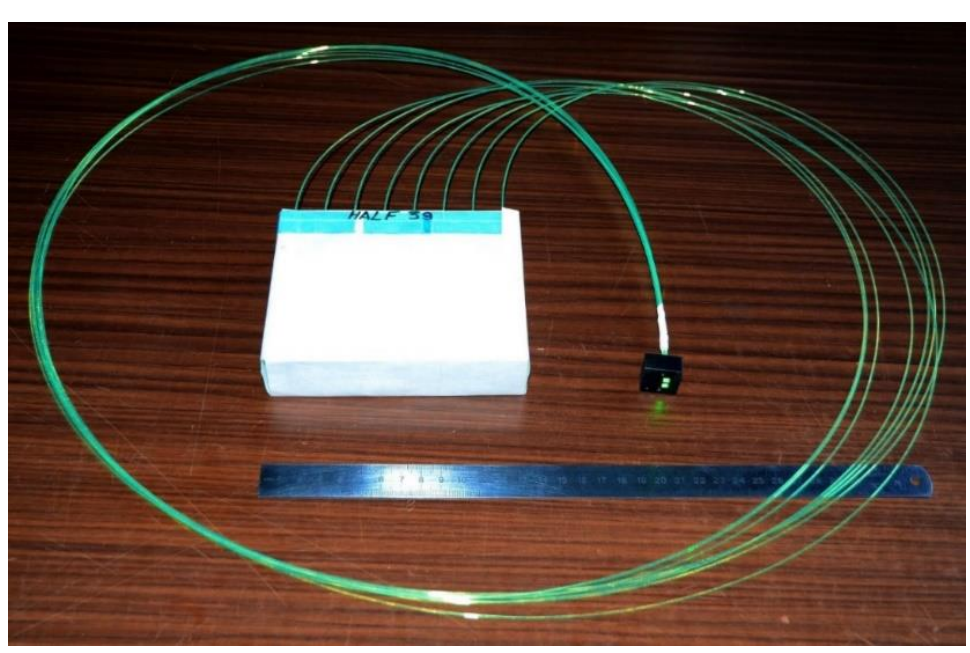

Fig. 1. The prototype half tile made to measure the light output and time resolution. 
of the beam tests allow us to proceed with full-scale production of tiles for the CHOD.

\subsection{The samples under test.}

The samples of differenThe larget thickness $(7-30 \mathrm{~mm})$ have been made in the shape of bars and plates (tiles) with the areas of $25 \times 80,108 \times 134$ and $108 \times 268 \mathrm{of} \mathrm{mm}^{2}$. The length of the WLSfibers was $0.5 \mathrm{~m}$ and $1.5 \mathrm{~m}$ respectively. Scintillator composition is $2 \%$ p-Terphenyl $+0.05 \%$ POPOP. Besides the fast UV-scintillator doped with $2 \%$ of PBD was manufactured. Two types of SiPMs were applied, $3 \times 3 \mathrm{~mm}^{2}$ SensL MicroFC-30035-SMT-WP and $2.5 \times 2.5 \mathrm{~mm}^{2}$ MRS APD 150-50 (CPTA, Russia) [5]. Bias voltage was set at the maximum specified levels. Preamplifiers provide the gain in 10-40 range. QDC with 50 ns gate and TDC were used in the tests.

\section{Setup for cosmic tests}

The tiles were tested with cosmic muons using coincidence between two trigger counters $(\mathrm{C} 1$ and C2) of $10 \times 50 \times 60 \mathrm{~mm}^{3}$ size. The trigger counters were positioned perpendicular to each other above and below the tested samples so that muons hit the area of $10 \times 60 \mathrm{~mm}^{2}$ size. The trigger rate was about one count per six seconds. A typical cosmic spectrum is shown in Fig. 2 for a 3

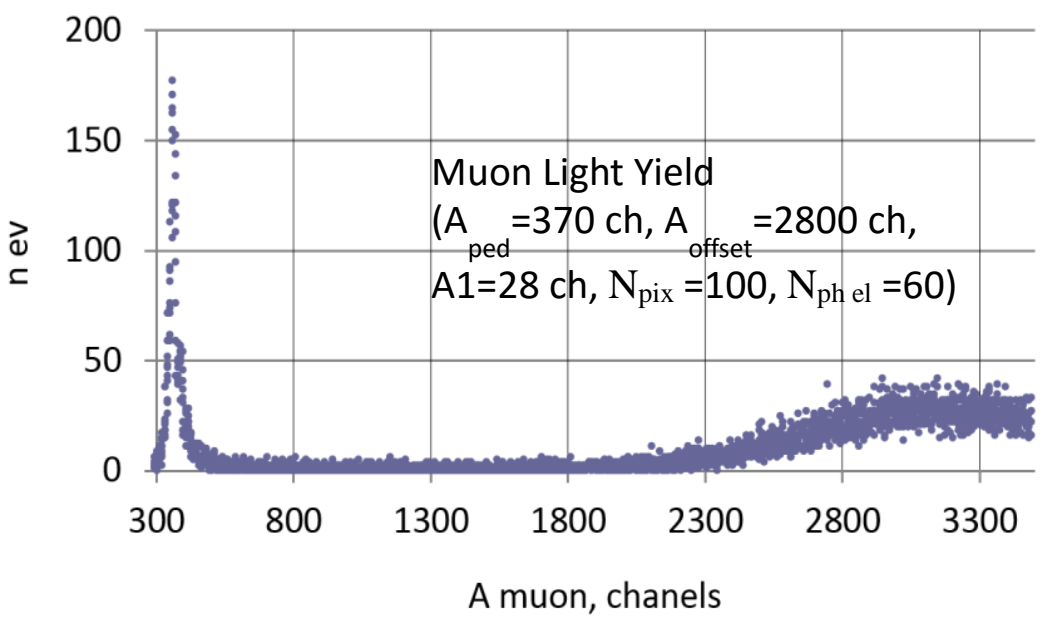

Fig.2. $\mathrm{N}_{\mathrm{pix}}$ - average number of fired pixels, $\mathrm{A} 1$ - single ph.e. charge, $\mathrm{N}_{\text {ph.el. }}$ - average number of ph.e.

cm thick sample.

\subsection{Calibration of detector readout channels.}

QDC scale calibration was performed with a light-emitting diode (LED). The amplitude spectrum for the case of small amount of light from the LED is presented in Fig. 3.

As a model function the convolution of the Poisson distribution and a set of Gaussians characterizing the operation of multiple pixels SiPM were used. The parameters of this function are determined by fitting of the experimental data with this analytical model (see magenta line in Fig.3). In practice, it is well known that the number of photo electrons is described by the Poisson distribution and the spectra of single-pixel shape close to a Gaussian. However, in the presence of the noise background we have to add a "substrate" (see. Fig. 3, the leftmost peak). As the parameters of fitting curve, the following quantities were used: the mean value of pedestal (PED) 
signal, the standard deviation of PED signal, Poisson distribution average value, the average value Fit position one pixel signal $\left(A_{1}\right)$, the average value of the experimental spectrum $\langle\mathrm{A}\rangle$, the standard deviation of the experimental spectrum (RMS). With these parameters we calibrated the QDC scale including the average number of fired pixels $-\left\langle\mathrm{n}_{\text {pix }}\right\rangle=(\langle\mathrm{A}\rangle-\mathrm{PED}) /$ $\mathrm{A}_{1}$ the number of recorded

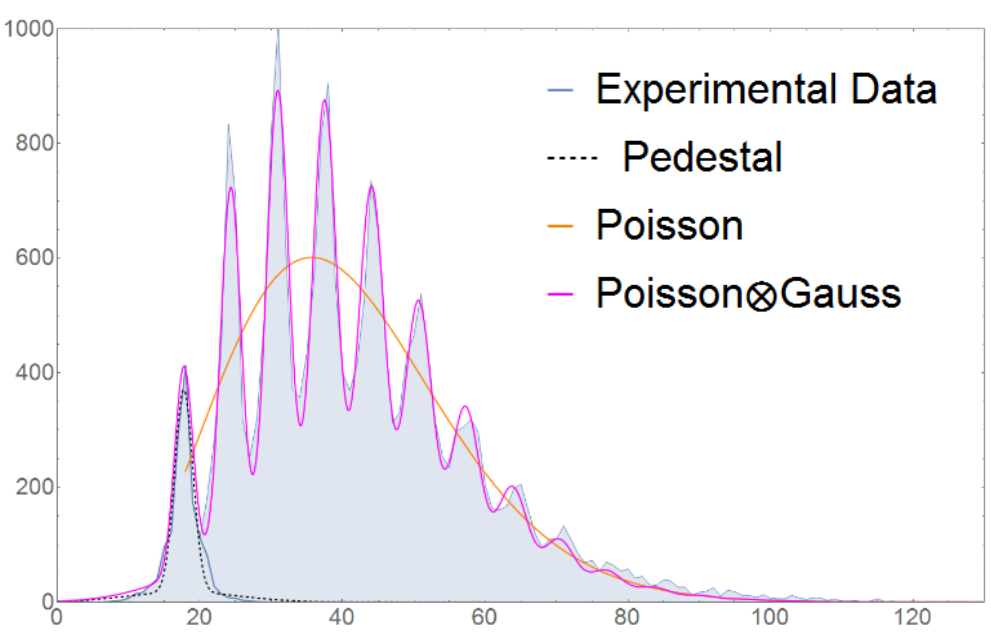

Fig. 3. The amplitude spectrum (experimental data) of the light emitting diode with a small amount of light. Colored curves show different model descriptions. photoelectrons $-\left\langle\mathrm{N}_{\mathrm{pe}}\right\rangle=(\langle\mathrm{A}\rangle$ $-\mathrm{PED})^{2} /\left(\mathrm{RMS}^{2}-\left(\sigma_{\mathrm{PED}}\right)^{2}\left\langle\mathrm{~N}_{\mathrm{pe}}\right\rangle=\ln (1 / P(0))\right.$, where $P(0)$ - probability for zero signal (pedestal). The values of fit parameters at different operating voltage of SiPM are shown in Fig.4

Light Yield (LY) calibrated in the average number of fired pixels and the number of recorded photoelectrons. The ratio determines the amount of cross-current of the neighboring fired pixels. The these relationships is observation for large over voltages of so-called crossinterference (crosstalk). Crosstalk should be taken into account, for example, when measuring the efficiency of detection is determined by Poisson statistics. These factors can contribute more

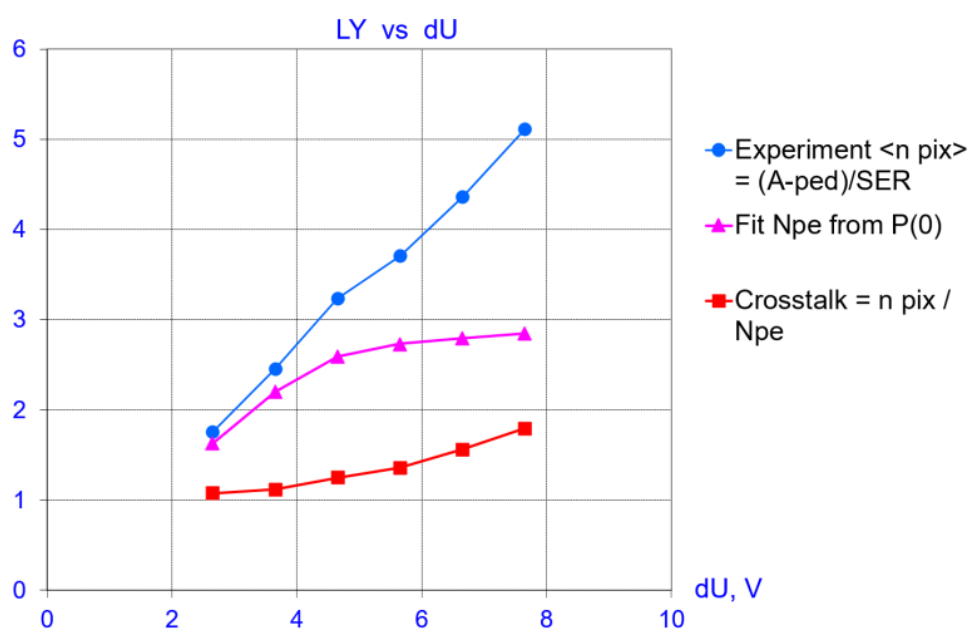

Fig.4. Light yield in fired pixels and ph.e. vs overvoltage. than $50 \%$ in the LY.

\section{Results}

\subsection{Light yield vs thickness.}

Fig. 5 demonstrates how LY depends on the scintillator thickness for the $25 \times 80 \mathrm{~mm}^{2}$ bars. Based on these data, the following conclusions concerning the LY can be made:

- The dependence of LY on the thickness of scintillator is nonlinear and is close to the square root of the thickness. This conclusion is also confirmed by the MC calculations.

- LY of the scintillators with Kuraray WLS Y11 multiclad fibers is nearly 1.6 times higher than with Saint-Gobain fast WLS multiclad BCF-92 fibers. 
- Diamond polishing (DMP) of the rough scintillator surface made by a milling machine gives $\sim 30 \%$ increase in LY compared to the non-polished surface.

- LY of UV scintillator read out by Kuraray scintillating multiclad fiber SCSF-78 is matching to a standard scintillator read out by Saint-Gobain WLS single-clad fiber $\mathrm{BCF}-91$.

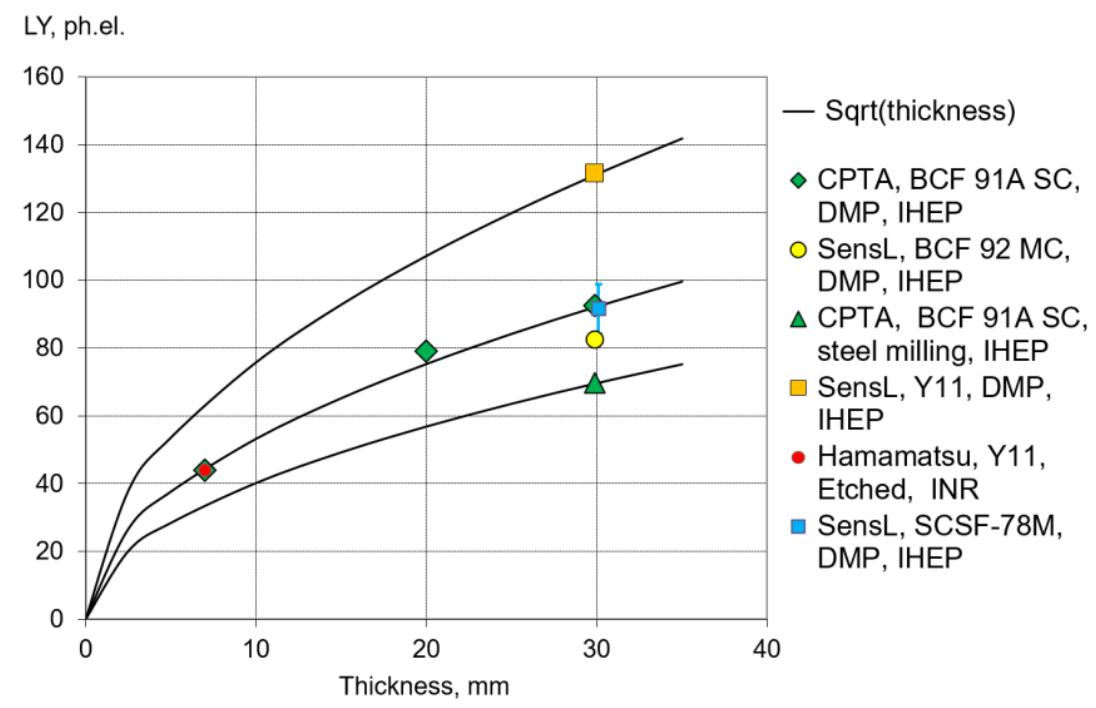

Fig.5. LY vs scintillator bar $\left(25 \times 80 \mathrm{~mm}^{2}\right)$ thickness, SiPM and WLS-fiber types and surface quality of scintillators (DMP, steel milling) and its origin. SC - single clad, MC - multi clad.

Scintillator LY vs the half-size tile thickness $\left(108 \times 134 \mathrm{~mm}^{2}\right)$ is shown in Fig. 6. Light yield for the full-size tile read out with fast BCF-92 fibers is also shown. The picture shows: the number of fibers, the type and length of the WLS fiber, step of fibers, the transverse dimension of the tile, surface condition and sample number.

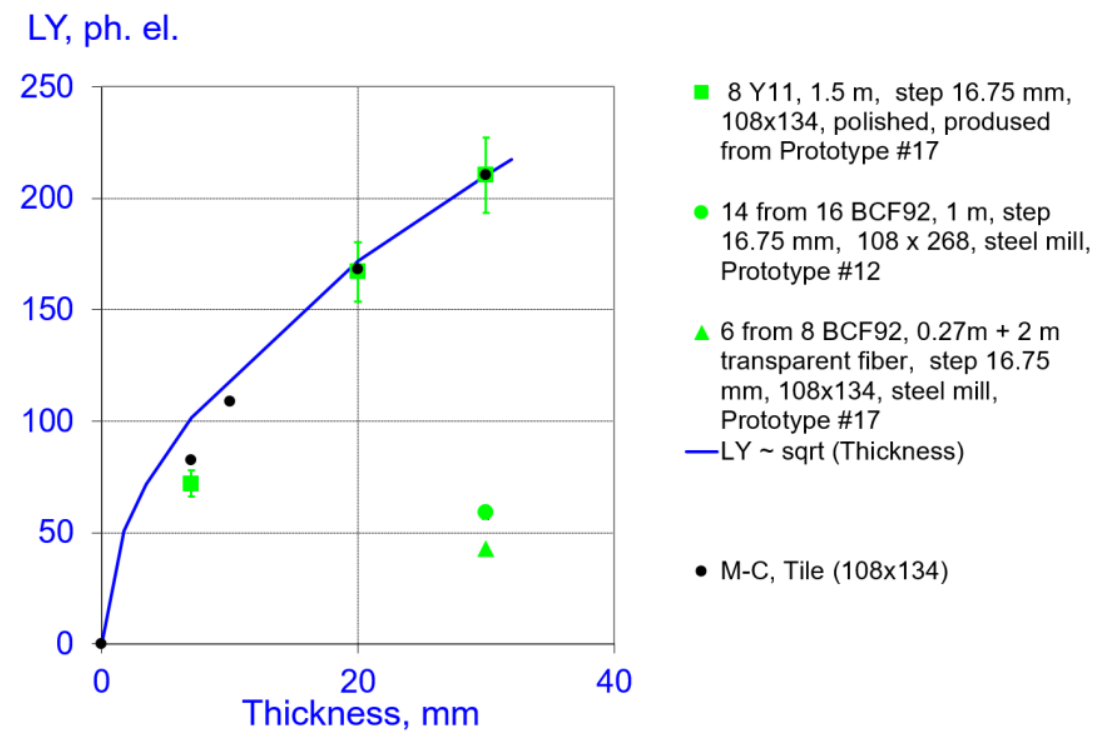

Fig. 6. Scintillator LY vs thickness: green markers - experimental points, the black markers - MC results.

The nonlinearity observed in MC calculations is confirmed by the full-size tile experimental data. Results presented in Fig.6 were obtained by removing the scintillator thickness with milling 
machine from one measurement point to another. LY of the scintillators with Y11 fibers readout is nearly 4 times larger than an option with BCF-92.

\subsection{Time Resolution and Delay Time vs Threshold.}

The results of timing measurements of the studied elements are shown in Fig. 7. Y-axis shows the time resolution and the time stamp offset depending on the discriminator threshold. Time resolution is characterized by the value of the standard deviation of the timing spectrum of Gaussian fit (Sigma T). The time stamp offset in its turn represents the shift of the peak position of the Gaussian fit (Shift Time - Delta T). We used low-threshold discriminator (Leading edge Discriminator - LeD) and compensated discriminator (Constant Fraction Discriminator - CFD). The time resolutions were obtained for light yields shown on the Fig. 5 and 6.

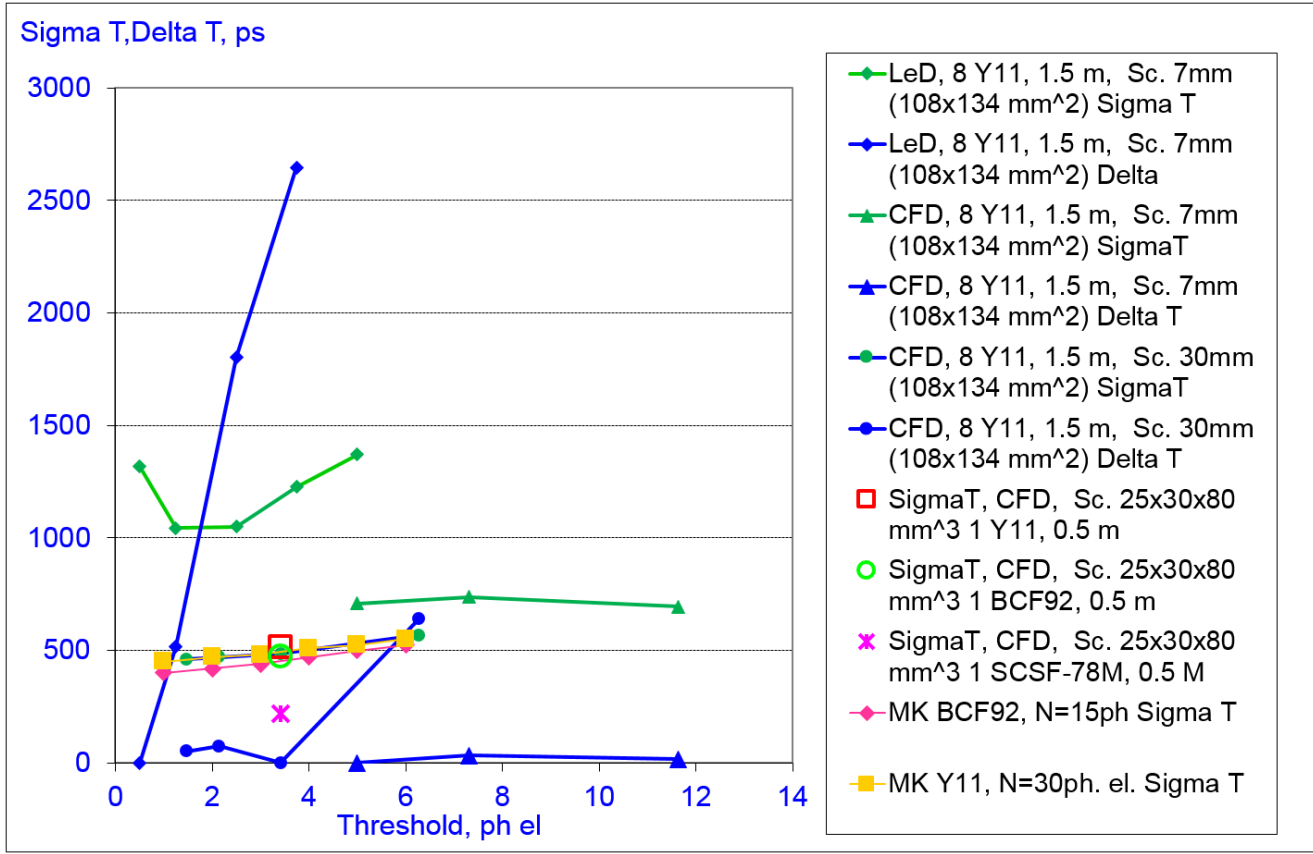

Fig. 7. The time characteristics of the studied elements vs discriminator threshold (see text).

From Fig. 7 one can conclude the following:

- the use of LeD instead of CFD has two drawbacks: first - a worse time resolution due to the input signal amplitude fluctuations; second - a significant time shift of the output signal at different thresholds, which may affect the event detection efficiency;

- the scintillators with Y11 and BCF92 fibers readout give the same time resolution (about $0.5 \mathrm{~ns}$ ), which is also confirmed by Monte Carlo simulation, although Y11 has much slower decay time ( $\sim 8 \mathrm{~ns})$ than BCF-92 $(2.7 \mathrm{~ns})$;

- the best result $(0.22 \mathrm{~ns})$ of the time resolution with the MIP detection is obtained for the pair of UV scintillator and SCSF-78M scintillation fiber used as WLS.

\section{Conclusions}

- Cosmic tests were done at IHEP to obtain the light yield and timing for polystyrene scintillator detectors with readout by WLS fibers BCF91A, BCF92, Y11 (Kuraray), and 
reemitting scintillating fiber SCSF-78M (Kuraray). SiPMs $3 \times 3 \mathrm{~mm}^{2}$ MicroFC-30035SMT-WP (SensL, Ireland) and 2.5×2.5 $\mathrm{mm}^{2} 150-50$ (CPTA, Russia) were used.

- The samples of different thickness $(7-30 \mathrm{~mm})$ in the shape of bricks and plates with the areas of $25 \times 80$ and $108 \times 134(268) \mathrm{mm}^{2}$ respectively have been measured. Besides samples of "ordinary" scintillator with additions of $2 \%$ p-Terphenyl $+0.05 \%$ POPOP, the rapid ultraviolet scintillator with single $2 \%$ dope of PBD was used.

- It is shown that the dependence of LY on the thickness of scintillator is nonlinear and close to the power function with square root, which is also confirmed by the calculations carried out by Monte Carlo.

- LY of the scintillators with Y11 fibers readout is nearly 2 times larger than with BCF-92.

- Our measurements and MC simulation show that the scintillators with Y11 and BCF92 fibers readout give the same time resolution (about $0.5 \mathrm{~ns}$ ), although Y11 has much slower decay time ( $~ 8 \mathrm{~ns})$ than BCF-92 $(2.7 \mathrm{~ns})$.

- The best result ( $0.22 \mathrm{~ns})$ of the time resolution with the MIP detection is obtained for the pair of ultraviolet scintillator and SCSF-78M scintillation fiber used as WLS.

- The LeD applied instead of CFD has worsened the time resolution and caused significant time walk.

- Crosstalk should be taken into account, for example, when measuring the detection efficiency, which is determined by Poisson statistics. These factors can contribute more than $50 \%$ in the LY.

\section{Acknowledgements}

We are grateful to V.F. Obraztsov, I.Mannelli, Yu.G.Kudenko and V.N. Bolotov for their permanent interest and support of our study; G.I. Britvich, E.N. Gushchin, S.A.Kholodenko, V.P.Sugonyaev, M.V.Medinskiy and O.Mineev for their useful discussions, help in organizing and operating the test benches; A.M.Kleschov, V.V.Volkov,M.E.Sandomirskiy and K.G. Kachnov for the high skilled execution of optical and mechanical tasks.

This work was performed under the Contract number N.4h.44.90.13.1118 from 31.05.2013.

\section{References}

[1] F. Hahn et al., The NA62 Collaboration, Technical Design, NA62-10-07, (2010) (http: //na62. web. cern. ch/NA62/Documents/TechnicalDesign.html).

[2] S.A. Kholodenko, A.A. Khudyakov, I. Mannelli, V.F. Obraztsov, V.D. Samoylenko, V.K. Semenov and V.P. Sugonyaev. Time resolution measurements of scintillating counters for a new NA62 trigger charged hodoscope. (2014) JINST 9 C09002.

[3] V. Rykalin, V. Brekhovskikh, S. Chernichenko, A. Gorin and V. Semenov. Development of the Polystyrene Scintillator Technology and Particle Detectors on their Base. Journal of Physical Science and Application 5 (2015) 6-13.

[4] http://sensl.com/products-page/bseries/microfb-300xx-smt/

[5] http: //www. cpta-apd.ru/PDF/CPTA $\% 20 M R S-A P D \% 20$ avalanche $\% 20$ photodiode 20en150-50.pdf 